\title{
Assessment of Robot Guidance Modalities Conveying Instructions to Humans in Emergency Situations
}

\author{
Paul Robinette* ${ }^{*}$, Alan R. Wagner ${ }^{\dagger}$, Ayanna M. Howard* \\ ${ }^{*}$ School of Electrical and Computer Engineering \\ Georgia Institute of Technology \\ ${ }^{\dagger}$ Georgia Tech Research Institute \\ Atlanta, GA, USA \\ probinette3@gatech.edu, alan.wagner@gtri.gatech.edu, ayanna.howard@ece.gatech.edu
}

\begin{abstract}
Motivated by the desire to mitigate human casualties in emergency situations, this paper explores various guidance modalities provided by a robotic platform for instructing humans to safely evacuate during an emergency. We focus on physical modifications of the robot, which enables visual guidance instructions, since auditory guidance instructions pose potential problems in a noisy emergency environment. Robotic platforms can convey visual guidance instructions through motion, static signs, dynamic signs, and gestures using single or multiple arms. In this paper, we discuss the different guidance modalities instantiated by different physical platform constructs and assess the abilities of the platforms to convey information related to evacuation. Human-robot interaction studies with 192 participants show that participants were able to understand the information conveyed by the various robotic constructs in $75.8 \%$ of cases when using dynamic signs with multi-arm gestures, as opposed to $18.0 \%$ when using static signs for visual guidance. Of interest to note is that dynamic signs had equivalent performance to single-arm gestures overall but drastically different performances at the two distance levels tested. Based on these studies, we conclude that dynamic signs are important for information conveyance when the robot is in close proximity to the human but multiarm gestures are necessary when information must be conveyed across a greater distance.
\end{abstract}

\section{INTRODUCTION}

Emergencies such as building fires, tornados, terrorist attacks and active shooter scenarios happen with little warning and require quick response from the affected individuals to maximize survival. As robots become more popular in daily life, they can be used as dynamic, embodied guidance aids for victims of these emergencies. Our previous work has shown that in-situ robots can improve existing technology, such as static emergency exit signs and alarms, by communicating the conditions of the emergency site to command posts while finding and guiding victims of the emergency out of danger [14], [13], [17]. Robot guidance can adapt to conditions inside the site to prevent crowding at exits or guide victims away from dangerous areas. Robots can also provide guidance in emergencies that require sheltering in place or moving to a safe location inside a building; responses that are not currently handled by signs in most buildings.
Prior research indicates that conveying guidance information to a small percentage of evacuees drastically improves survivability [16]. In other work, we have found that robots are generally accepted and trusted by humans in simulated emergency situations but the trust levels dropped considerably when participants in the experiments were unable to determine what the robot was instructing them to do [15]. Building on those results, in this paper we explore various visual guidance modalities deployed on mobile robot platforms and their effect on human understanding of guidance instructions.

Three categories of visual methods for conveying guidance information were identified: static signs, dynamic signs, and arm gestures. These categories were combined with each other and a mobile robot base to form five different platforms with information conveyance packages and one baseline platform with no specialized information conveyance abilities.

The information conveyance ability of these robots was tested by recording simulations of the six platforms performing each of four guidance instructions at both an instruction point near the victim and a point further away from the victim. Human participants then interpreted the instructions and thus rated the understandability of the information being conveyed.

\section{RELATED WORK}

Considerable research has focused on using robots for search and rescue applications. Bethel and Murphy studied how volunteers reacted to rescue robots in a simulated urban disaster [2], [9]. They created several recommendations for how robots should approach, contact, and interact with the victims. For the approach and other motions, the researchers suggest using smooth acceleration and deceleration. In contrast, typical robots are usually jerky when moving in an unknown environment. The researchers also suggested using blue lighting around the robot to convey a sense of calm. For interaction, they note that there are several different "zones" where the robot can be: the intimate zone ( 0 to 0.46 meters), the personal zone ( 0.46 to 1.22 meters), the social zone (1.22 to 3.66 meters) and the public zone (further than 3.66 meters). Robots are assumed to stay in the social zone or closer. To 
communicate, the researchers assumed that the robots would have to be in the intimate or personal zones. They suggested using voice communication to reassure the victim and music when there is no information to communicate. More recent work has extended this to UAVs [6].

Simulated emotions have also been tested to see how it can improve human responses when a robot instructs a human to leave a room due to an unexpected emergency [8]. This work began by using videos posted online to determine if humans could understand the emotions being displayed by the robot [12]. The robot gave clear, verbal instructions aided by emotional actions, so participants were only tested on their ability to understand the robot's emotional actions and comply with its requests. Studies in non-verbal robot communications have found that robots and humans work better in teams when the robot performs non-verbal cues and gestures during the interaction [3]. Robots have also been commanded by human hand gestures [18].

Orkin and Roy were inspired by early chatbots, such as ELIZA, to create a game to simulate interactions, via the internet, between two people in a restaurant [10]. Users would join the game and randomly be assigned as either a waiter or a client. Then they would proceed to interact as if they were in an actual restaurant. The researchers noted that users typically took the game seriously and acted as if they were in a real social situation. The experiment generated considerable data related to responses to typical prompts in the environment. Users were solicited through blogs, web postings, emails and social media. A total of 3,355 users played 5,200 games over several months and completed a survey afterwards. Other research has expanded on this crowdsourced data gathering process to help train a robot for a space mission [5]. The simulation sets up two users on a Mars base, one as the robot and one as the astronaut.

\section{RobOt TO HUMAN INFORMATION CONVEYANCE MODALITIES}

This work is focused on methods to convey instructions to victims in a potentially noisy emergency situation. The most useful instructions in an emergency are directional: either instructing victims to go to a particular location or instructing them to stay in place. At this point, we only consider a victim standing in one location observing a robot giving instructions, so the simplest set of instructions with the largest utility is: 1) proceed to the left or right (we arbitrarily chose left in all cases), 2) proceed forward, 3) turn around, and 4) stay in place. Each modality allowed different actions to be presented for the four different instructions given to the participant. The instructions as conveyed by each modality are given below.

\section{A. Mobile Platform}

A simple mobile platform can convey information even when not equipped with specialized displays and actuators. For this work, we assume that the mobile platform is nonholonomic but otherwise a fully controllable ground robot. For directional instruction (left, forward, turn around) the robot

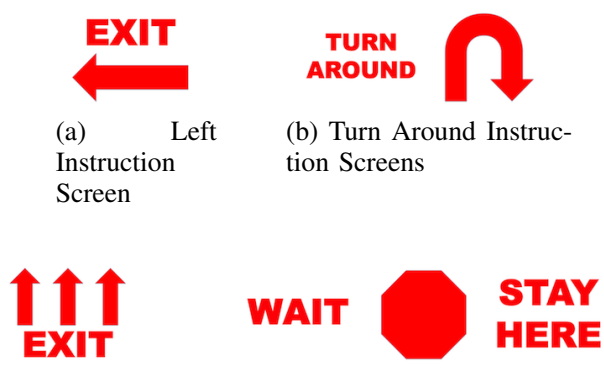

(c) Forward Instruction

Screen

Figure 1: Dynamic Signs Text and Symbols

first turns in the direction it wishes the human to proceed and then oscillates about that direction by 30 degrees left and right. In this way it can point in the general direction that the human should proceed but still indicate that information is being displayed through action. To instruct the human to stay in place, the robot spins in place.

\section{B. Static Sign}

In [15], we mounted a static sign consisting of an arrow and the word "Exit" to a holonomic platform and pointed the robot in the direction of the exit. This was both confusing to the participants and unrealistic on actual platforms, so for this experiment the static sign simply consists of information giving the intent of the robot. The words "Emergency Guide Robot" were displayed on the static signs.

\section{Dynamic Sign}

A dynamic sign gives the robot the ability to convey situation-dependent information including arrows, text, and animations. Such a sign can consist of a tablet or computer monitor or even a set of LEDs. For the purposes of this work, we assume that the sign will give sufficient resolution such that it can show English words and simple symbols. For the left and forward directions, the dynamic sign shows the word "EXIT" and an arrow or set of arrows that point in and grow along the direction of the instruction (either left or forward, see Figures 1a and 1c). To indicate that the participant should turn around, the sign alternates between the u-turn symbol and the text "TURN AROUND" (Figure 1b) For the stay in place instruction the sign cycles through three screens: "WAIT," "STAY HERE," and a red octagon (Figure 1d).

\section{Arm Gestures}

Arm gestures are frequently used by humans in many different contexts, from police officers guiding cars to airport personnel directing aircraft to parents guiding children. Arms also provide the ability to attract attention at a distance by waving. We developed gestures for robots equipped with a single arm or multiple arms. For these purposes, we assume that the arms have at least two degrees of freedom: base rotation and at least one bend. 


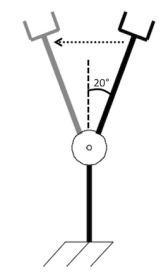

(a) Example of a Single Arm Waving

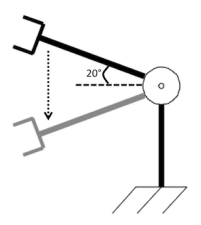
Single Arm Pointing

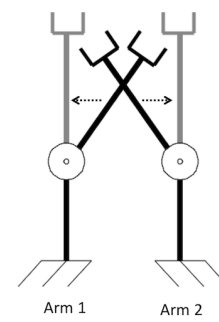

(c) Example of Multiple Arms Crossing (b) Example of a

Figure 2: Examples of Arm Gestures. In each case, the arm moves from the solid black position to the solid gray position in the direction of the dotted arrow.

Attention is attracted by a platform with a single arm by holding it upright and waving it horizontally 20 degrees left and right (Figure 2a). For directional instructions, the whole platform turns to face the direction it wishes the human to proceed and the arm points forward (Figure 2b). The arm then oscillates slightly along the vertical axis to "wave" the participant in the required direction. For the stay in place instruction the robot faces the participant and waves its arm in the same manner it used to attract attention from this stationary position.

Multi-arm gestures are very similar to the single arm gestures in directional instructions: two arms wave in the direction in which the participant should proceed. For the stay in place instruction, the robot faces the participant and alternates between both arms straight up and arms crossed (Figure 2c). Attention is attracted by waving upright arms.

\section{E. Hypotheses}

We hypothesize that a simple mobile platform will be unable to provide clear guidance instructions to humans but the addition of information conveyance devices will allow for increasing understandability. The static sign is not expected to provide any specific guidance information but the dynamic sign is expected to produce significantly better results for the near case where text and symbols will be legible. For the far case, the arm gestures are expected to produce significant increases in clarity, with multi-arm gestures being more understandable than single arm gestures. Finally, we hypothesize that a combined approach featuring a dynamic sign and multiarm gestures will convey guidance information best at both distance levels.

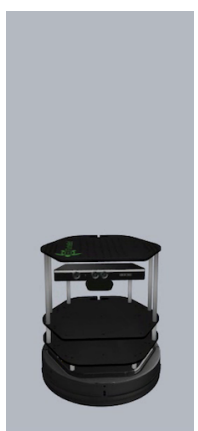

(a) Baseline

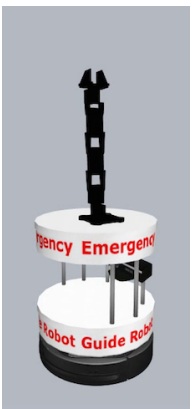

(d) Single Arm Gesture

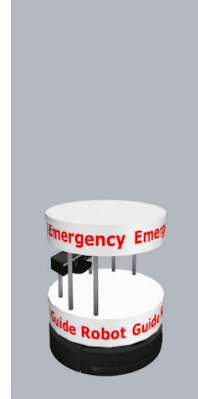

(b) Static Sign

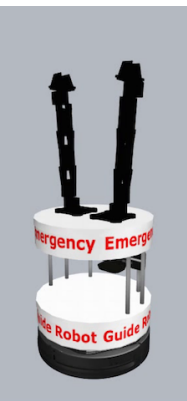

(e) Multi-Arm Gesture

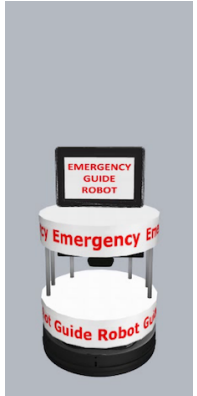

(c) Dynamic Sign

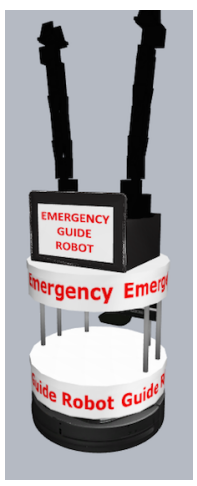

(f) Multi-Arm Gesture with Dynamic Sign
Figure 3: Robot Guidance Platforms

\section{RoBot PlatForms}

To test our hypotheses we developed platforms based on each of the information conveyance modalities above as well as one platform that combined several modalities. We started with a Baseline robot (Figure 3a) to test the mobile platform instructions. We then created the Static Sign platform (Figure $3 \mathrm{~b})$ to determine if these signs produced any differences from the Baseline. All further platforms used static signs as well as other modalities. A Dynamic Sign platform (Figure 3c) as well as both Single Arm Gesture (Figure 3d) and Multi-Arm Gesture (Figure 3e) platforms were developed to test each of those modalities alone. Two arms were selected for the multiarm platform to be as close as possible to human gestures. A final platform combined a dynamic display with multi-arm gestures to fully test our hypothesis (Figure 3f).

\section{INFORMATION CONVEYANCE DEVICES}

\section{A. Mobile Platform}

All robot platforms were based off of the Willow Garage Turtlebot 2 due to its ease of use and general availability. The Turtlebot 2 is a $42 \mathrm{~cm}$ tall platform with a Kobuki base, a netbook running ROS for control and a Microsoft Kinect for sensing. The Tutlebot used in this experiment was simulated with 3D models of all components. This platform 
was tested without modification to determine the baseline understandability of guidance instructions.

\section{B. Static Sign}

All robots except the Baseline carried signs that declared the robot's purpose as an emergency guidance aid. The signs were in two cylindrical components: one on the top of the Turtlebot and one covering the netbook just above the base. The top sign displayed "Emergency" in each of the four cardinal directions around the cylinder and the bottom sign displayed "Robot Guide" in the same manner.

\section{Dynamic Sign}

An 11" Samsung Galaxy Tab was used as the dynamic sign. The tablet was mounted upright on top of the Turtlebot in landscape orientation. The tablet displayed instructions to the user in a combination of arrows, stop-signs and English words.

\section{Gesture Arms}

The PhantomX Pincher AX-12 arm was used in all platforms that required arms. This arm has five degrees of freedom and a maximum reach of $35 \mathrm{~cm}$. For the Single Arm Gesture platform, the arm was mounted to the center of the top of the Turtlebot. For the Multi-Arm Gesture platform, the arms were mounted on the left and right sides of the top of the Turtlebot. For the Multi-Arm Gesture with Dynamic Sign platform, the arms were mounted as in the Multi-Arm Gesture platform but on a box approximately $12 \mathrm{~cm}$ high such that no arm gesture would collide with the display.

\section{EXPERIMENTAL SETUP}

To evaluate human understanding of the robot guidance modalities we utilized a between-subjects experiment. Participants were recruited and the study conducted using Amazon's Mechanical Turk service. Other studies have found that Mechanical Turk provides a more diverse participant base than traditional human studies performed with university students [11], [4], [1], [7]. These studies found that the Mechanical Turk user base is generally younger in age but otherwise demographically similar to the general population of the United States (at the time of those studies, Mechanical Turk was only available in USA). A total of 192 participants performed this survey.

Participants began the study by reading and acknowledging a consent form. Next, they completed a demographic survey collecting information about gender, age, nationality (Mechanical Turk is currently available for residents of both USA and India), occupation, and education. Then, the participants were presented with videos of one particular robot performing each of the four instructions (one instruction for each video). A victim's ability to understand visual displays of guidance information depends on the distance between the victim and the display. For this reason, robots were tested at both a near and a far distance (see Figure 7 for the layout). Each participant was only shown the videos for one robot at one distance level. For each video, participants indicated which

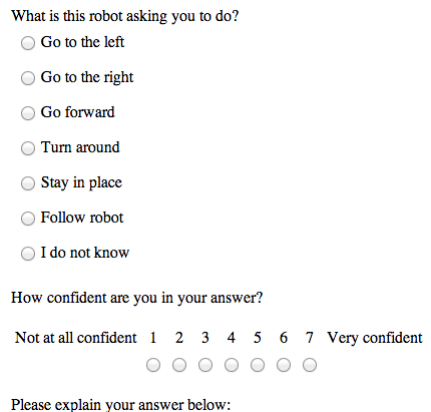

Figure 4: Questions Asked for Each Video

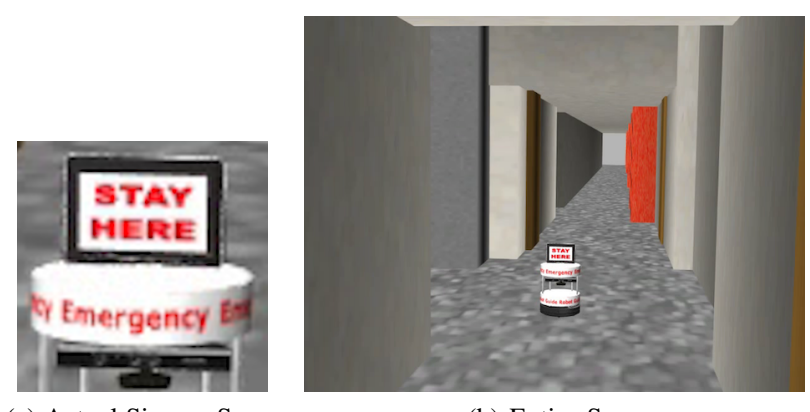

(a) Actual Size on Screen

(b) Entire Scene

Figure 5: Dynamic Sign Platform at Near Instruction Point Displaying Wait Instruction

instruction they thought was being performed, estimated their confidence in that answer (a number 1 through 7), and gave an explanation for their answer (see Figure 4 for the exact questions and layout). Several instructions were given as multiple choice answers for each video, including some that never appeared in the test so that participants could not use process of elimination to give an answer. The dependent variable being measured was their answer to the multiple choice question and the comments were used to understand that answer. The order of the videos was randomized. The videos were each between 15 and 19 seconds long. Each video was 800 x 600 pixels in size. Participants were paid $\$ 0.50$ for completing the survey. IRB approval was obtained before the study began.

Videos of the instructions were created in the Unity Game Engine. The videos were hosted on YouTube and embedded into the survey form on Mechanical Turk. Each commercially available component of each platform was simulated using CAD files provided by the manufacturer. These components were assembled into robot platforms in the Blender 3D modeling software and imported into Unity for simulation. Custom components, such as the signs on the robot, were created in Blender and Unity.

The testing environment was a long hallway with open areas (potential exits) immediately to the left of the camera view, at the far end in front of the camera, and behind the camera. Screenshots of the robot in the near and far positions can be found in Figures 5 and 6, respectively. A map of the environment can be seen in Figure 7. 


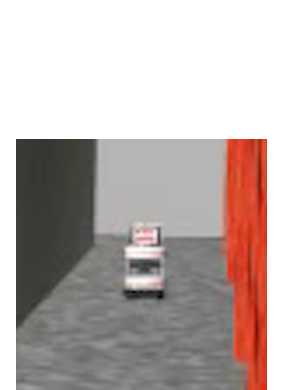

(a) Actual Size on Screen

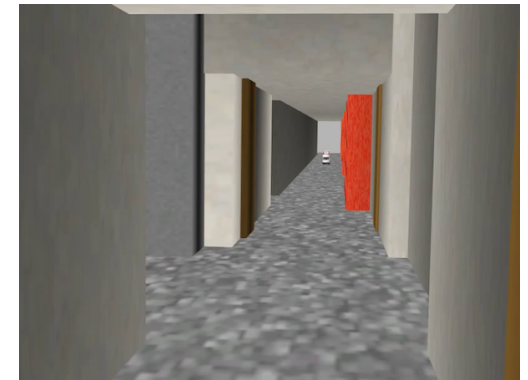

(b) Entire Scene
Figure 6: Dynamic Sign Platform at Far Instruction Point Displaying Wait Instruction

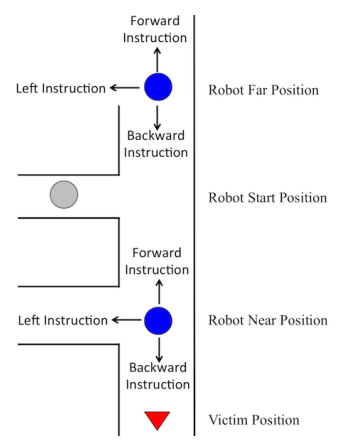

Figure 7: Map of Testing Environment

Sixteen participants viewed each robot at each distance level. No participant was allowed to perform the experiment more than once, so participants did not have any information about the other robots or their actions.

\section{RESULTS}

In general, adding features to the baseline platform improved understandability of instruction (Figures 8 and 9). As expected, the Multi-Arm Gestures with Dynamic Display platform had the best overall understandability $(75.8 \%$ overall) but, unexpectedly, the Static Sign platform performed worse than the Baseline (18.0\% and $28.1 \%$, respectively). Unfortunately, the confidence values reported by the participants had no consistent base and thus could not be used to give insight into the results. Some participants ranked their confidence with a high number on the Likert scale but indicated uncertainty in their comments, others did the opposite.

We expected that there would be little or no difference between the Baseline and Static Sign platforms; however, the results show that the Baseline performed considerably better than the Static Sign for the left instruction at the near distance. Based on comments, it seems that participants were able to infer the rotation of the robot by the position of the Kinect. As such, since the top sign on the Static Sign platform partially obscured the Kinect, participants were not able to observe any orientation of the robot. The other results are very similar between the two robots. Participants were unable to see which direction the robot was pointing for the near distance forward

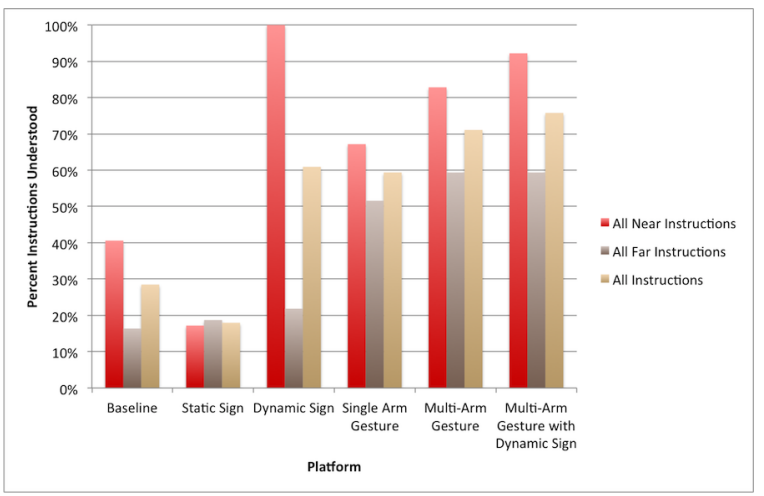

Figure 8: Percent Instructions Understood at Each Distance Level and Overall by Platform Type

Table I: Pairwise Chi-Squared Results Comparing Guidance Instruction Modalities (p-Values)

\begin{tabular}{|c|c|c|c|c|c|}
\hline Platform & Baseline & $\begin{array}{c}\text { Static } \\
\text { Sign }\end{array}$ & $\begin{array}{c}\text { Dynamic } \\
\text { Sign }\end{array}$ & $\begin{array}{c}\text { Single } \\
\text { Arm } \\
\text { Gesture }\end{array}$ & $\begin{array}{c}\text { Multi- } \\
\text { Arm } \\
\text { Gesture }\end{array}$ \\
\hline Static Sign & 0.054 & & & & \\
\hline $\begin{array}{c}\text { Dynamic } \\
\text { Sign }\end{array}$ & $<0.001$ & $<0.001$ & & & \\
\hline $\begin{array}{c}\text { Single } \\
\text { Arm } \\
\text { Gesture }\end{array}$ & $<0.001$ & $<0.001$ & 0.798 & & \\
\hline $\begin{array}{c}\text { Multi-Arm } \\
\text { Gesture }\end{array}$ & $<0.001$ & $<0.001$ & 0.086 & 0.049 & \\
\hline $\begin{array}{c}\text { Multi-Arm } \\
\text { Gesture } \\
\text { with } \\
\text { Dynamic } \\
\text { Sign }\end{array}$ & $<0.001$ & $<0.001$ & 0.011 & 0.005 & 0.396 \\
\hline
\end{tabular}

and backward instructions, even when the Kinect was not obscured. For the far condition, participants in the surveys for both of these robots indicated that they could not see any understandable action. Many guessed that they should follow the robot (an option included in the survey even though it was not shown as an instruction). They reasoned that the robot moved away from them and waited, thus indicating that they should proceed in that direction. Overall, results from the Baseline and Static Sign platforms did not show statistical difference in a Chi-Squared test $(p=0.054)$. There were, however, significant differences between both of these robots' understandability and every other platform $(\mathrm{p}<0.001)$ (Table I).

As expected, the Dynamic Sign platform performed very well for the near instructions. Every participant indicated that the screen simply told them what to do and thus the answer was easy. The distance for the far instructions was specifically chosen such that a tablet or other screen about this size could not be clearly read from that instruction position. Each participant in the far survey confirmed that they were unable to discern any instruction from the Dynamic Sign platform, even when the large red octagon was displayed. Examples of the Dynamic Sign platform at near and far distances in 


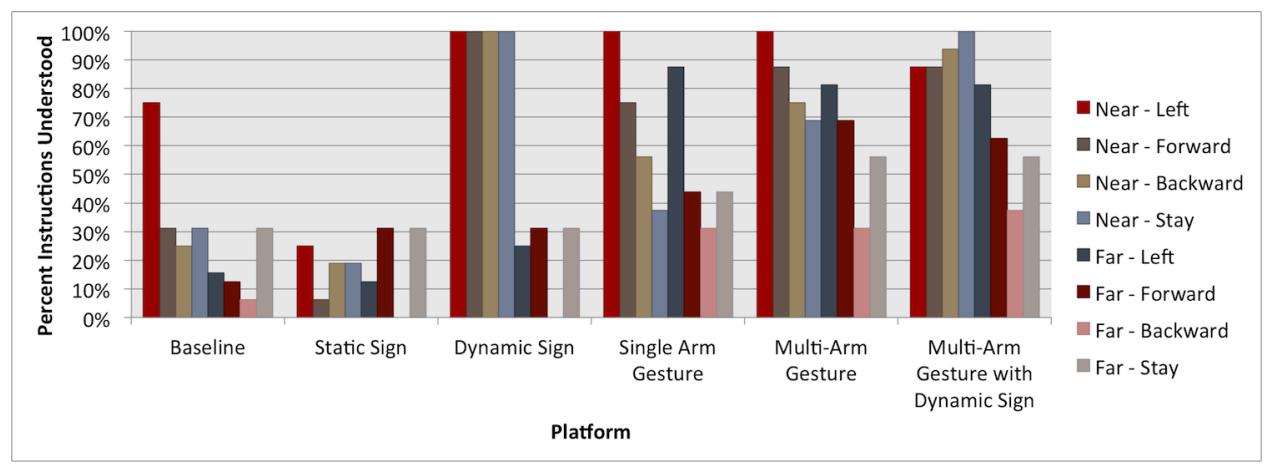

Figure 9: Percent of Instructions Understood by Platform Type

Figures 5 and 6, respectively, show that participants in the far case were at a great disadvantage here. Recall that participants only viewed instructions from one robot at one distance level, so no participants were able to observe an instruction while close to the robot and then recognize it from a distance. A majority of the participants did not even realize that a display of any type was mounted on the platform. They wrote that there was simply an indecipherable red light on top. As in the previous cases, confused participants tended to assume that they should follow the robot if they could not understand a particular instruction. Overall, this platform was significantly different from the Baseline, Static Sign and Multi-Arm Gesture with Dynamic Sign (Table I).

The Single Arm Gesture platform performed about as well at the Dynamic Sign platform overall $(59.4 \%$ and $60.9 \%$, respectively, $\mathrm{p}=0.798$ ) but had much lower variance between the near and far conditions. This indicates that guidance robots should be equipped with at least one arm unless the environment is conducive to individuals reading a screen. Participants had difficulty determining which direction the arm was pointing when it was giving forwards or backwards instructions. This difficulty increased with distance. Some of the difficulty could have been an artifact of the simulation. Participants also had difficulty understanding the stay instruction in both near and far cases. This is because the single arm is not able to articulate any standard stop gesture. In addition to the previously reported statistical results, the Single Arm Gesture platform had statistically significant differences between the Multi-Arm Gesture ( $p=0.049)$ and Multi-Arm Gesture with Dynamic Display $(\mathrm{p}=0.005)$ platforms (Table I).

The Multi-Arm Gesture platform solved the problem with forwards, backwards, and stay gestures by adding a second arm to provide instructions in the same style as airport ground crews. This produced an overall understandability of $71.1 \%$ of instructions. There was some confusion still with the forward and backward commands that was also exacerbated with distance, but comments indicated a greater confidence with the answers chosen. The stay instruction was confusing to some but most still recognized it as indicating to not proceed in that direction, even if they did not understand that they were supposed to stay in place. Otherwise, confusion generally resulted in the participant choosing the follow option.

Surprisingly, the Multi-Arm Gesture with Dynamic Display platform had no statistically significant differences from the Multi-Arm Gesture platform ( $\mathrm{p}=0.396)$. Overall, $75.8 \%$ of its instructions were recognized correctly. We expected the near results to be identical to the Dynamic Sign results, but comments from two participants lead us to believe they confused the robot's reference frame with the camera's reference frame and thought that the robot was indicating right instead of left and backward instead of forward. In those two cases the robot also turns such that the tablet cannot be seen after the robot arrives at the instruction point, which might have increased the confusion. The robot performed as expected at the far distance level. Overall, after accounting for qualitative results gleaned from the comments, the combined approach of using a dynamic display and multi-arm gestures produced the best results for both near and far conditions.

Recruiting participants through Mechanical Turk did not seem to have a major effect on the results. Most participants took the survey seriously and gave considered, thoughtful comments for each question. Some indicated frustration when they were unable to understand the robot. One even requested that the participants receive training on robot gestures if we would like the results to be improved. There was some confusion as to exactly what the robot arms were, but the participants ability to understand the instructions did not depend on whether they referred to the arms as "antennas", "cranes" or even "tentacles." Only one participant gave bizarre answers, writing "I believe that the robot is trying to say that the walls are dirty and [that] they need to be cleaned." and "The reason I chose [to] follow [the] robot is because I think that the robot is attempting to communicate with the human." for two different questions. Those results were still included for completeness.

A total of 122 males and 69 females participated in the experiment (one participant declined to give his or her gender). Gender was not found to have a significant effect on the results $(\mathrm{p}=0.183)$. Participants spanned all education categories with a majority indicating that they had at least some college experience. This, too, was found to not have a significant effect on the results $(\mathrm{p}=0.758)$. Most participants reported that they were in their 20s but 10 were over 50 years of age, so the age 
range in this study is likely much more broad than would be found in testing on a college campus. Occupations spanned a wide range. We grouped them into the following categories for analysis: self-employed, technical, customer service, clerical, unemployed and other. Neither occupation nor age were found to have a significant effect on the results $(\mathrm{p}=0.441$ and $\mathrm{p}$ $=0.446$, respectively). There was not enough variability in nationality to test for statistical differences. Expected results for Chi-Squared tests were calculated by taking an average of the results of all platforms weighted by the number of participants in that demographic who participated in that survey.

Across all robot platforms the backward instruction was understood the worst $(39.6 \%)$ and the left instruction was understood the best $(65.6 \%)$. The instruction did have a significant effect on the results $(\mathrm{p}<0.001)$ but all instructions were tested for each robot, so the results are still valid.

\section{CONCLUSIONS AND FUTURE WORK}

Our survey explored the capability of different robotic platforms in instructing humans to find a safe exit in an emergency. We focused on visual guidance to avoid potential problems with audio instructions in a noisy emergency environment. Platforms were varied by adding signs to indicate function, a tablet to display instructions in written language or recognizable symbols, and an arm or arms to gesture to the victim.

Through quantitative and qualitative results we found that a ground platform with a dynamic display and multi-arm gestures provides the clearest instructions to victims in an emergency. The addition of single arm gestures or a dynamic display by itself also performed considerably better than an unmodified ground robot. A surprising result provides a word of caution to fellow roboticists: adding seemingly trivial aesthetics such as signs can produce differences in outcomes of human-robot interaction experiments.

These results were obtained by soliciting volunteers on Amazon's Mechanical Turk service. As a consequence, our participant pool was significantly different from traditional experiments with university students. We maintain that these results are more general than on-campus studies but it must be noted that the Mechanical Turk population is mainly composed of young adult Americans with above average education.

These tests all focused on a wheeled ground platform with modifications. Despite other differences, the Multi-Arm Gesture platform is likely to be very similar to a humanoid robot in terms of understandability of guidance instructions given that the action set is very similar. Nevertheless, this assertion should be tested. We also plan to perform real-world tests to verify that the simulations presented here are reliable indicators of understandability.

These results will prove valuable when designing any sort of automated guide. When combined with audio instructions, the Multi-Arm Gesture with Dynamic Sign platform could be used as a source of information in malls, a tour guide in museums, or a director of traffic in city streets, as well as a source of guidance for humans during emergencies. Dynamic signs can also be used to customize instructions for nearby humans to overcome language and cultural barriers. Arm gestures remove the need for language in communication and provide larger motions that help when communicating with confused or even panicked humans.

\section{AcknOWLEDGEMEnts}

Partial support for this research was provided by the Motorola Foundation Professorship.

\section{REFERENCES}

[1] Adam J Berinsky, Gregory A Huber, and Gabriel S Lenz. Evaluating online labor markets for experimental research: Amazon. com's mechanical turk. Political Analysis, 20(3):351-368, 2012.

[2] C. L. Bethel and R. R. Murphy. Survey of non-facial/non-verbal affective expressions for appearance-constrained robots. IEEE Transactions on Systems, Man, And Cybernetics Part C, 38(1):83-92, 2008.

[3] Cynthia Breazeal, Cory D Kidd, Andrea Lockerd Thomaz, Guy Hoffman, and Matt Berlin. Effects of nonverbal communication on efficiency and robustness in human-robot teamwork. In Intelligent Robots and Systems, 2005.(IROS 2005). 2005 IEEE/RSJ International Conference on, pages 708-713. IEEE, 2005.

[4] Michael Buhrmester, Tracy Kwang, and Samuel D Gosling. Amazon's mechanical turk a new source of inexpensive, yet high-quality, data? Perspectives on Psychological Science, 6(1):3-5, 2011.

[5] S. Chernova, J. Orkin, and C. Breazeal. Crowdsourcing hri through online multiplayer games. AAAI Fall Symposium 2010, 2010.

[6] Brittany A Duncan and Robin R Murphy. Comfortable approach distance with small unmanned aerial vehicles. In RO-MAN, 2013 IEEE, pages 786-792. IEEE, 2013.

[7] John Joseph Horton and Lydia B Chilton. The labor economics of paid crowdsourcing. In Proceedings of the 11th ACM conference on Electronic commerce, pages 209-218. ACM, 2010.

[8] Lilia Moshkina. Improving request compliance through robot affect. In AAAI, 2012.

[9] R. R. Murphy. Human-robot interaction in rescue robotics. IEEE Transactions on Systems, Man, and Cybernetics, Part C Applications and Reviews, 34(2):138-153, 2004.

[10] J. Orkin and D. Roy. The restaurant game: Learning social behavior and language from thousands of players online. Journal of Game Development (JOGD), 3(1):39-60, 2007.

[11] Gabriele Paolacci, Jesse Chandler, and Panagiotis Ipeirotis. Running experiments on amazon mechanical turk. Judgment and Decision Making, 5(5):411-419, 2010.

[12] Sunghyun Park, Lilia Moshkina, and Ronald C Arkina. Recognizing nonverbal affective behavior in humanoid robots. Intelligent Autonomous Systems 11: Ias-11, page 12, 2010.

[13] P. Robinette and A.M. Howard. Emergency evacuation robot design. In ANS EPRRSD - 13th Robotics \& Remote Systems for Hazardous Environments and 11th Emergency Preparedness \& Response, 2011.

[14] P. Robinette and A.M. Howard. Incorporating a model of human panic behavior for robotic-based emergency evacuation. In RO-MAN, 2011 IEEE, pages 47-52. IEEE, 2011.

[15] P. Robinette and A.M. Howard. Trust in emergency evacuation robots. In 10th IEEE International Symposium on Safety Security and Rescue Robotics (SSRR 2012), 2012.

[16] P. Robinette, P.A. Vela, and A.M. Howard. Information propagation applied to robot-assisted evacuation. In 2012 IEEE International Conference on Robotics and Automation, 2012.

[17] Paul Robinette, Alan R Wagner, and Ayanna M Howard. Building and maintaining trust between humans and guidance robots in an emergency. In 2013 AAAI Spring Symposium Series, 2013.

[18] Michael Van den Bergh, Daniel Carton, Roderick De Nijs, Nikos Mitsou, Christian Landsiedel, Kolja Kuehnlenz, Dirk Wollherr, Luc Van Gool, and Martin Buss. Real-time 3d hand gesture interaction with a robot for understanding directions from humans. In RO-MAN, 2011 IEEE, pages 357-362. IEEE, 2011. 